\title{
An Intelligent and Object-oriented Blueprint for a Mobile Learning Institute Information System
}

\author{
Emre Erturk \\ Eastern Institute of Technology, Napier, New Zealand
}

\begin{abstract}
The purpose of this conceptual paper is to develop an original framework that summarizes the applicable software model, and guiding approaches and principles for designing a comprehensive information system for a mobile learning institute. Artificial intelligence has played a role in various strands of computer-mediated education, including e-learning learning platforms. In adaptive delivery mode, learning applications simulate the behavior and interaction of human teachers. AI functionality can be incorporated into mobile learning because mobile devices have the processing power and operate in wireless networks. In addition, the individual elements of the framework are illustrated in industry-standard Unified Modeling Language diagrams. A review of educational and artificial intelligence literatures shows how these fields are related and helps identify techniques to develop smart mobile learning applications. An overview of mobile learning application design helps build a theoretical foundation in this area. The individual parts of the proposed framework are: the architectural framework, cognitive framework, project definition framework, application design framework, and organizational framework. The organizational framework lays the conceptual foundation for creating and implementing an information system within a newly established mobile learning institute. This paper fills a gap in the literature as there is currently no comprehensive overview of this topic. Furthermore, there are currently no existing schools, organizations, or individual departments within that focus only on and provide mobile learning. In addition to fostering the development of such a mobile learning institute, the proposed framework is also useful for guiding technical work on content design.
\end{abstract}

\section{Introduction}

The first goal of this paper is to discuss the role of artificial intelligence (AI) in online education, specifically the use of AI in so-called m-learning. Mlearning uses mobile computing devices such as smartphones and tablets. There is a growing push from online educators to make online learning content suitable for mobile devices. Blended learning in higher and university education is a mixture of learning environments, combining the face-to-face classroom with computer-mediated online education. Adaptive learning is the use of computers as interactive devices similar to the behavior of dynamic human teachers. The natural synthesis of these two trends will lead to the incorporation of artificial intelligence concepts into new mobile learning applications.

This paper starts with a brief review of computermediated and adaptive education. Next, the paper develops a conceptual framework using high-level diagrams for a sample adaptive learning application. The scope of this framework is software that may be used in asynchronous, online, and computermediated distance learning activities (in other words e-learning and m-learning). In turn, this framework is composed of subordinate frameworks including the architectural framework, the cognitive framework, the project definition framework, and the application design framework.

The final piece of this framework, i.e. the organizational framework, provides a foundation for creating and implementing an information system within a newly established mobile learning institute.

Unified Modeling Language (UML) is a standardized general-purpose object-oriented modeling language with a rich palette of diagrams for different levels of analysis and implementation. The original diagrams used in this paper adhere generally to UML notation. The use of UML is an additional way in which this paper makes an original contribution to academic and technical literature on education and artificial intelligence.

Another benefit of UML is that there are already software development tools available for mobile devices that are model driven (such as IBM Rational Rhapsody), i.e. that can generate code from models [15]. Furthermore, there are rapid application development (RAD) and Visual Design tools available [13]. The smart aspects of certain applications are the higher degree of interaction with the user and the greater adaptivity by providing relevant feed-back and feed-forward to different 
users. These aspects can be developed with the help of multimedia content and sophisticated user interfaces with various options.

\section{Review of literature}

With the implementation of more capable hardware and faster networks, the trend in online education is to provide smart blended delivery that accommodates students with different learning styles and goals [2]. Artificial intelligence (AI) techniques can be used in education in innovative ways. First, computer tools can be used to model and simulate how experts or teachers think about a subject. Second, computer tools can monitor students and ascertain how they are approaching and learning the subject. These ideas can be combined and they offer potential to improve instructional systems and increase the quality of student learning.

One of the early examples of AI in education has been computerized grading of student assessments. This is faster than manual grading, and provides timelier feedback to students. Another example of AI in computer-mediated learning is appropriately tailoring content to individual students. This is especially useful in subjects where there are multiple correct (and incorrect) ways to solve problems. Depending on the student's answer and personal solution method, smart instructional software supplements the student's learning accordingly. This adaptive learning functionality can be accomplished by software components that include a visual agent to interface with the student and a background algorithm to guide the learning activity. This guidance requires a number of sub-functions. One of these includes accumulating knowledge about each student by interpreting the clues picked up during interactions with the student. The knowledge base should in itself also continue to adapt as its student population grows. Thus the learning platform will improve its instructional techniques.

Much of the current effort among online educators focuses on making blended or distant learning content suitable for mobile devices. This is especially true for content that involves communication and interaction with students [9]. Therefore the natural trend will be to incorporate philosophical and practical concepts from artificial intelligence into mobile application design. Recent versions of mobile operating systems are largely open source or have open source components. Furthermore, many development tools and existing applications are open source and/or freeware for the public. This environment allows more public discussion and more public contribution to the design of new learning applications. For example, Pocatilu prescribes some steps for developing mobile learning (m-learning) content and suggests types of content for a basic m-learning platform such as quizzes, tests, homework assessments, forums, and a messaging component. The communities where new learning applications can be distributed and tested are typically at educational institutions and online open source platforms [14].

\section{Architectural Framework}

Most mobile learning applications are deployed according to the three-tier client-server architecture. Most of the literature presumes this to be the case, and authors tend to focus on other aspects of application design instead of the computer networking perspective [11]. In the three-tier software architecture, the user interface, the processing logic, and the data management operate independently. The parts of the application related to each tier can be developed separately and can run on separate devices. It is necessary to document this general consensus in a clear way. The presentation tier displays information to the user and captures user input. A common form of this is a web-based graphical user interface that is deployed via internet browsers and standard communication protocols. The logic tier controls the application's functional flow and performs detailed processing. The data tier consists of database servers that store, retrieve, and manipulate data. The UML deployment diagram below summarizes the three-tier architecture specifically for adaptive learning applications and mobile devices.

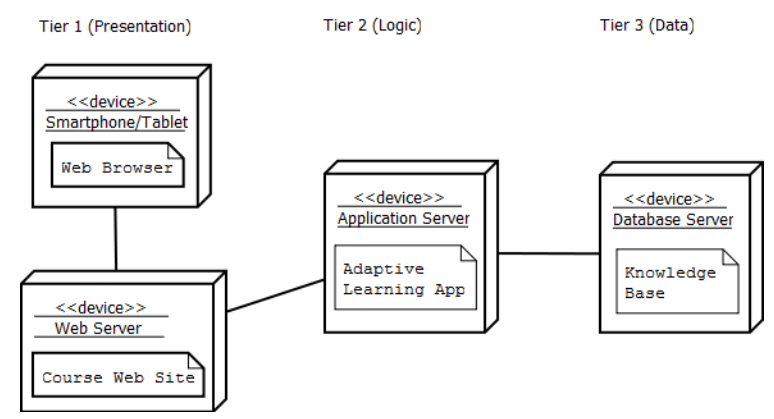

Figure 1. Adaptive Mobile Learning Application Deployment Diagram

\section{Cognitive Framework}

An alternative perspective on education is to analyze it as being made up of memes, i.e. units of cultural information such as beliefs, trends, imitations, images, replications, behavior, and so on [12]. Memes are also covered in artificial intelligence literature in order to depict and simulate the evolution of cultures. Successful memes spread well, and are adapted because they capture fundamental human needs at a fundamental level at a subconscious level and help people communicate and satisfy their needs. Examples of currently 
popular educational attitudes (or memes) are: "learning must be fun" and "learning must be applicable to one's future job." Distance and blended learning have led to new education memes and paradigms in recent years [1]. One of these is that students are builders of content for themselves rather than just consumers. They produce information that may help the learning process of other students. Elearning provides an advantage here by capturing and archiving student input into learning platforms.

Reasoning (inference) engines and cognitive agents will reach even higher standards of facilitating more personalized learning, assisting toward individual tutoring, and enabling student-guided learning pathways. While doing this, the social context of traditional education needs to be preserved to a certain degree in e-learning and m-learning so that group learning activities can also take place online. Educational memes will continue to evolve, and understanding them and foresight into upcoming trends is important for long-lasting effective educational content development.

In artificial intelligence literature, skeuomorphs have been addressed because they are important part of human users' way of thinking. They are defined as "material metaphors instantiated through our technologies" [6]. Digital skeuomorphs are visual objects that resemble the original physical objects in order to help by making the underlying function more familiar and appealing to the typical user. Even the word 'tablet' is a skeuomorph to describe a flat touch-screen mobile computer larger than a phone, and alludes to the clay, wax, or wooden tablets used as writing and drawing media in early civilizations. Skeuomorphs are commonly used in computer interface design through commonly used icons (such as buttons and folders). They are often necessary for describing or labeling new hardware and software objects. As a result, the application developer needs to consider ways to use them effectively while designing learning application interfaces.

A new generation functional programming language known as the Bluespec Codesign Language is used in developing mobile and embedded applications by describing algorithms 'as a set of rules' rather than instructions [7]. This language allows the designer to specify the hardware-software interface for the mobile device in the source code and assign tasks to either hardware or software depending on whichever is more suitable for performance or battery use. This independence allows software developers to focus better on artificial intelligence aspects than before. Furthermore, there are numerous open source development tools and online communities, for example, EulerGUI [16] and FreeHAL [17], specifically for artificial intelligence applications and using popular programming tools.

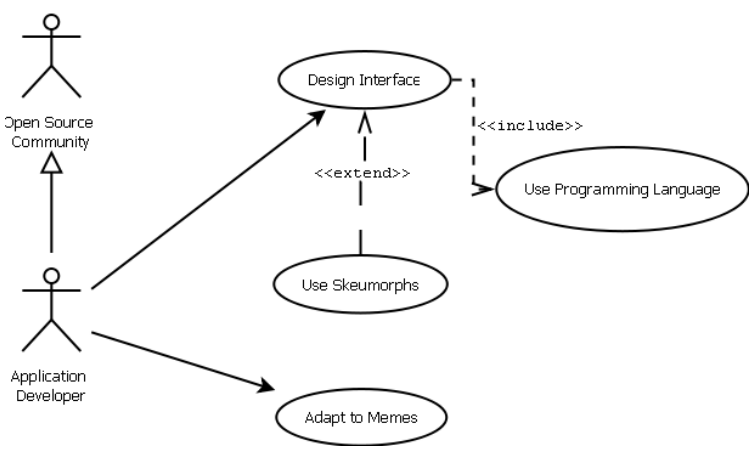

Figure 2. Mobile Learning Interface Development Use Case Diagram

\section{Project Definition Framework}

The design of new learning resources requires is in itself a project that requires a certain methodology for guiding the teamwork and the software development. The role of a methodology is to structure, plan, and document the development project. Many sophisticated project management methodologies have been formulated in the past (http://infolific.com/technology/methodologies/).

Therefore this paper will only review those nuances that pertain to learning content design projects and the project definition phase.

An example of a complete methodology to be used for educational design projects is by the Columbia University Center for New Media and Teaching [3]. There are many other educational design methodologies that apply generically to faceto-face and online courses. On the other hand, there are several unique concerns for mobile learning applications that should be discussed during project definition and initiation:

1. Mobile learning resources are often adaptations of existing online content to make them suitable for mobile devices. However, smartphones and tablets differ from larger computers in terms of touch-screen capability and being used anywhere. These must be taken into account for the design project. Participating in this type of learning and using the various technologies are skills in themselves for many blended learning students. The same goes for m-learning; the mobile platform experience should be proposed as a benefit.

2. A project involves timelines and costs. A justification for investing in m-learning is that education needs to keep up with technological advances. In addition, the course content becomes more enjoyable and effective, enhancing customer satisfaction. Using open source development tools will improve cost-effectiveness [14].

3. Mobile devices rely on kinesthetic and visual interfaces with the user as well as voice interaction. Different students also have different learning styles such as visual learning, auditory learning, and 
kinesthetic learning. Therefore new content will include multimedia, including animations, slides, and graphs [8]. The development of multimedia is time intensive. Editing and testing multimedia inevitably calls for additional staffing for a design project. If prototype screenshots can be produced, they will clarify project objectives and expected outcomes.

4. Designing a successful product requires gathering information to discover and satisfy the underlying human needs of the users [8]. There are two approaches to interface design. The first is a formal specification of what actions the user (i.e. the student) performs while doing a task, and listing and quantifying these actions in order to create a mechanical flow for the application. The second approach is ethnographic, and involves interviewing and observing software users to gain insights about their true motives. The second approach is necessary for discovering alternative ways to perform learning tasks and creating original interface formats.

\section{Application Design Framework}

An artificial intelligence application needs database files to store concepts and the relationships between those concepts (i.e. the semantic network) in order to acquire knowledge and continue to adapt. An expert system includes rules that define how human syntax and other input are interpreted and rule that define how concepts can be combined within an academic subject [4].

Intelligent software agents are programs that act with some degree of autonomy, are broader goal oriented, and can react to the environment in more flexible ways. A teaching agent is expected to assess student learning and student work, and to be able to provide genuine feedback or hints [5].

Metacognition is knowledge about when to use particular strategies for teaching, and is also necessary for the underlying adaptivity. The UML component diagram shows the breakdown of a sample adaptive learning software application in terms of artificial intelligence.

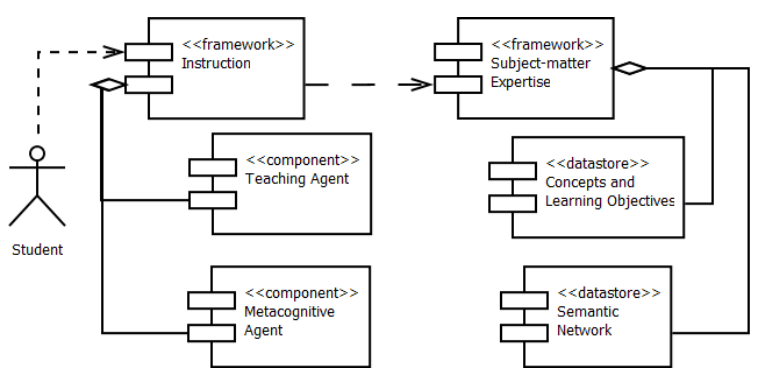

Figure 3. Adaptive Mobile Learning Application Component Diagram

\section{Organizational Framework}

Nano Mobile Learning Institute (NMLI) is a new hypothetical business, providing short courses and training to individuals and organizations around the world via m-learning (using smart phones and tablets), particularly focusing on business and computing skills. Furthermore, many of the courses are geared toward workplace training and on-the-job performance support.

The foundation of the new mobile learning institute consists of stating the mission, goals, and the organizational actors of the new enterprise. As such, the institute and its own information system will have nonfunctional requirements, i.e. its goals. The new institute needs to be: flexible in the variety of courses it provides both in New Zealand and internationally, provide a combination of good tuition pricing and high quality of course material, maintain a high degree of student satisfaction and retention, continually optimize its internal productivity and improve its own business processes, and strive for an industry-leading reputation.

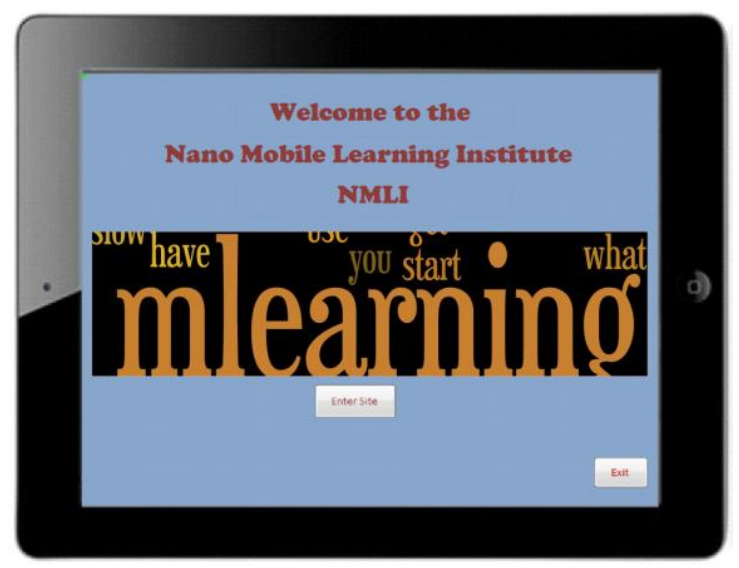

Figure 4. Learning System Prototype Screen

The following are the organizational users of the hypothetical learning institute information system:

1. The student user is anyone who takes courses provided by NMLI, including individual students and group enrollments through client companies.

2. The teacher users also serve as content managers as they create material for the courses.

3. The administrator is responsible for maintaining data such as enrolments, course schedules, customer inquiries, and archives.

4. The accountant is responsible for accounts payable, accounts receivable, and payroll.

5. The human resources specialist is concerned with the employment of new staff and the further training of current staff.

6. The CEO and other executives are interested in the performance and growth of the business. 
7. The marketing specialist is responsible for promotions and customer relations.

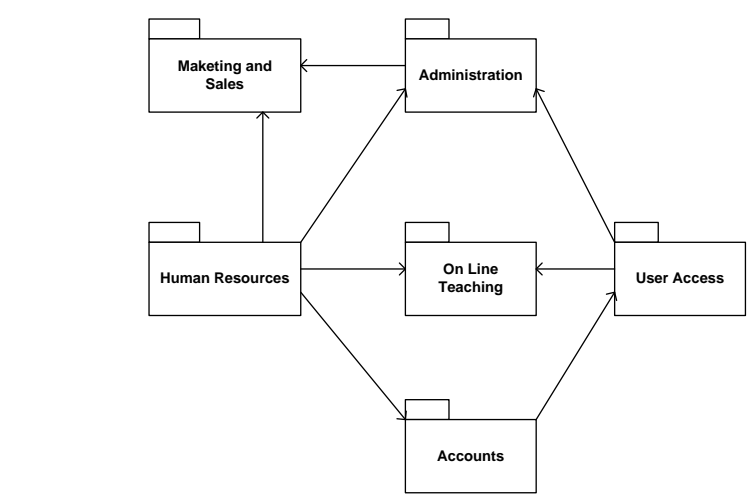

Initial Architecture Diagram

\section{Figure 5. Mobile Learning Institute Subsystems}

The new system will be made up of six subsystems:

1. The User Access subsystem is responsible for everyday user related tasks such as login, course downloads, test submissions and grades retrieval.

2. The On Line Teaching subsystem is the heart of the course delivery aspect of the system. It contains all information pertaining to users, courses, tests and grades. Users have limited direct access to this subsystem as their main access is via the User Access subsystem. This system is mainly used by teachers and administrators to create courses and course material. Managers can obtain general reports on courses via this subsystem.

3. The Accounts subsystem is responsible invoicing users, receiving payments, paying bills, and paying employees. This also involves the maintenance and security of accounting information. The only access the common user has is to their personal information. Management can use this subsystem to obtain financial reports.

4. The Human Resources subsystem covers hiring new employees, employee appraisals, employee assignments, discontinuation of employees, and regular maintenance of employee records.

5. The Administration subsystem handles the daily business of the organization such as adding, updating, and removing users and courses.

6. The Marketing and Sales subsystem is responsible for maintaining and growing the institute's customer base through campaigns, promotions, and regular contact with past and present clients. It also involves the commercial sale of multimedia items to customers online.

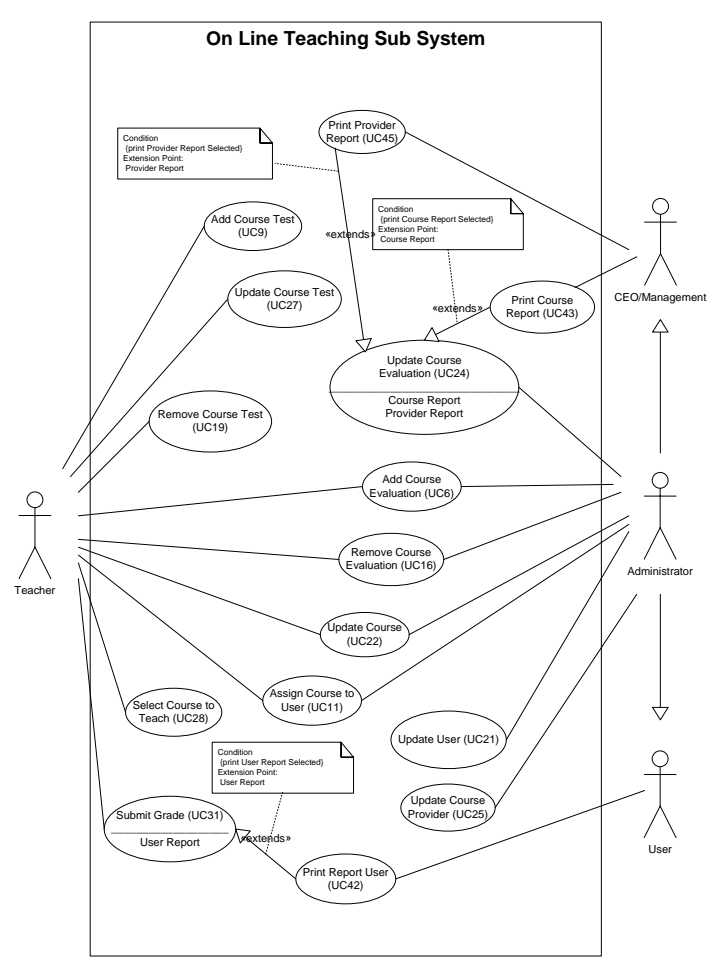
Figure 6. Use Case Diagram for the On Line
Teaching Subsystem

The design principles that are particularly important to an information system used by a mobile learning institute are functionality, reliability, security, flexibility, maintainability, and usability. Functionality suggests that students must enjoy course materials without being interrupted by technical or other problems. The software as presented on the user's mobile device must be reliable, that is, as content managers add course material, these need to be moderated to ensure consistency of quality and reliability of use.

NMLI will handle online payments and deliver confidential course results; therefore security (including user authentication and protection of confidential information) will be of great importance. Flexibility is also essential design features as the number of courses offered and the student population will grow and change with time. Any future upgrades to the system may be done via an object oriented approach by reusing, modifying, and extending classes within the program code. The system must be designed for easy future maintenance. A clear and well documented system will enable different individuals to be able to perform maintenance. 


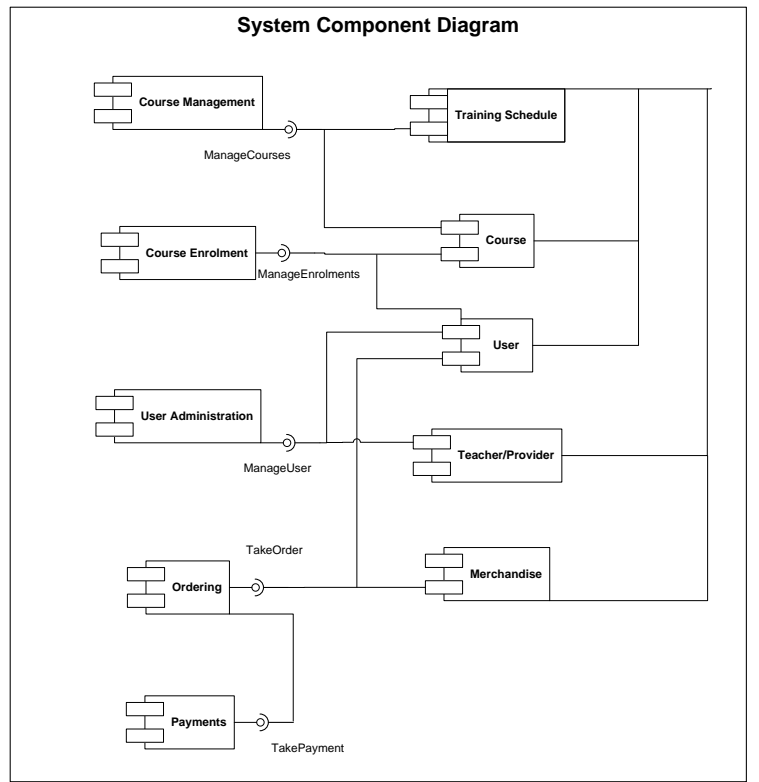

\section{Figure 7. Mobile Learning Institute Management Information System}

Usability means an excellent Human-Computer Interface design (HCI). The prototypes that have been designed to date demonstrate an intuitive and clear graphic user interface (GUI). The system will continue to use iterative agile techniques to ensure the system will continue to satisfy users.

The database design will be an object-relational database (ORD) that provides the features of an object oriented database while retaining some of the efficient data management functionality of a relational database. The nature of NMLI lends itself to an object oriented style of data storage particularly because complex data objects such as multimedia content are an important part of the business. This task involves digital curation, effectively cataloguing content and reviewing the ontology periodically. The database is also required to handle large volume of relatively simple data efficiently just like a relational database. The database will be compatible with operating systems such as Microsoft Windows, Linux, Mac OS X, and Unix-based systems.

A distributed database system is intended for this information system. The success of NMLI depends on its ability to reliably deliver its course content over the internet to mobile devices. A mobile replicated database system is suitable for web-based systems. The data will be replicated on the mobile device and stored as local data whenever there is a high-speed internet connection. When the data is updated locally, it is then synchronized back to the database servers, through a good internet connection. This will allow the user to download course material and catalogues and use them locally on the mobile device. It will also allow course progress or transactions to be made even if there are interruptions to the connection. It will provide a high degree of reliability and usability, independent of the quality of the user's internet connection.

Testing will consist of test sheets that are part of an overall test plan. The test sheets will show the plan for a given day or area, and then collect the test results. Black and white box testing will be carried out on all in-house software. System testing will take place in four stages:

1. Program level testing will be performed by software engineers by testing individual software classes and components where possible, or on a use case basis and then testing individual subsystems.

2. Alpha (verification) testing is also performed in an isolated (offline) environment by software engineers. This testing focuses on the whole system as one with many tasks and combinations. The object is to test for incorrect or unexpected results from incorrect or invalid inputs.

3. Beta (validation) testing is performed in a live environment. Teachers and employees from NMLI will be involved at this level to assess system performance and failure/recovery mechanisms.

4. Final Acceptance Testing is a formal series of tests and signs offs within NMLI. Any problems discovered must be referred back to at least the Alpha testing stage or the Program testing stage.

User manuals and full-technical documentation will be published on-line. The user training manuals will help new employees of NMLI learn to use the system. Downloadable user reference material will be made available to all users, including students. The training plan will be divided into departmental modules. General system training will be delivered formally to each department and further training will be geared toward the particular tasks of the department or individual employee. Each employee will be able to conduct on-line training from their workstation as necessary.

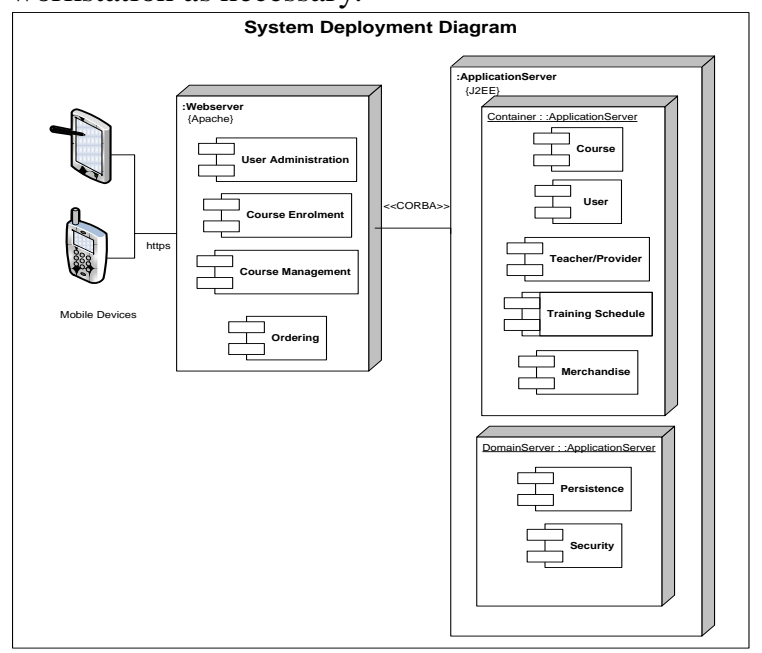

Figure 8. NMLI Information System: Sample Deployment Diagram 
Since NMLI is a new organization, there are no issues with changing over from an older information system. However, individual departments will be involved in their own separate testing and training so, in a sense, this will be a 'phased' launch. In the case of NMLI, a backup plan will be prepared and the necessary support will be on hand to correct any error or malfunction of the web services when the institute goes live for the first time. The internet support can be reduced to a nominal level once the institute is successfully operating.

\section{Conclusions and Recommendations}

First, this conceptual framework is useful for guiding technical work on content design. Second, it contributes to the theoretical literature in various areas such as mobile computing and e-learning. Smart learning applications need to be (and will be) used more in business and workplace training. Greater deployment of web-based training applications from a central server (which may also be cloud-based) will lead to long-run cost savings (in terms of labor and hardware) for those companies.

An important area that may be considered by future researchers is artificial intelligence incorporated into student group collaboration tools. Group work is a way to make individual students more active and help them learn together. Collaboration gives them experience working in teams that will be useful in their future jobs. Group collaboration is a key area in Web 2.0. Computermediated learning platforms will be able to generate automated groupings of students with similar interests, and suggesting resources and links to different groups of learners based on their interests.

There are currently no existing schools or organizations (or even individual departments within) that focus only on and provide mobile learning. There are many universities, schools, and businesses that have developed a mobile application just to help support their existing online or blended delivery. One of these is Cisco Systems, the leading manufacturer of networking equipment, and provider of related professional certifications. Their different mobile apps help deliver video, audio, and written content to two different types of audiences: anyone preparing to become certified in Cisco technologies, and Cisco employees themselves who are refreshing their knowledge or looking up solutions to technical problems they encounter in the field [19].

Micro-learning is growing in importance, especially in workplace training where the time for learning is constrained [18]. It utilizes small learning units flexibly depending on the context and time available, and gradually and purposefully builds macro knowledge. It can further be supported by appropriate preparatory and follow-up activities.
Micro-learning can be greatly enhanced through push communications on mobile devices as well as knowledge databases available online. Therefore, a well-organized micro-learning approach will play an important role for any institution that conducts $\mathrm{m}$ learning. In the long run, the experiences of a new mobile earning institute will provide fresh data and perspectives for future research on micro-learning.

\section{Acknowledgements}

The author would like to thank Mr. Marco Maiorana (MMaiorana@eit.ac.nz), graduate student and staff member (Business Analyst) at the Eastern Institute of Technology, for contributing Figures 4 through 8 (unpublished) as well as for providing his input into Section 7 as directed by the author.

\section{References}

[1] Andrews, G. R. (n.d.). Education Memes. http://home.earthlink.net/ bmgei/educate/ed_phil/edmemes.htm (Access date: 12 January 2012).

[2] Association for the Advancement of Artificial Intelligence (n.d.). Intelligent Tutoring \& Other Educational Uses of AI. http://aaai.org/AITopics/Education (Access date: 12 January 2012).

[3] Columbia University, Center for New Media Teaching and Learning (n.d.). Design Research. http://ccnmtl.columbia.edu/dr/spiral.html (Access date: 9 January 2012).

[4] Crowley, S. \& Medvedeva, O. (2003). A General Architecture for Intelligent Tutoring of Diagnostic Classification Problem Solving. Proceedings of the American Medical Informatics Association Annual Symposium, 2003, 185-189.

[5] Delgarno, B. \& Mills, C. (2007). A conceptual model for game based intelligent tutoring systems. Proceedings of the Australasian Society for Computers in Learning in Tertiary Education (ASCILITE) Annual Conference, 2007, 692-702.

[6] Gessler, N. (n.d.). Skeuomorphs and Cultural Algorithms. http://www.skeuomorph.com/ (Access date: 15 January 2012).

[7] King, M., Nirav D., \& Arvind, N. (2012) Automatic Generation of Hardware/software Interfaces. Proceedings of the seventeenth international conference on Architectural Support for Programming Languages and Operating Systems, ASPLOS '12, 325-336. (Access date: 27 January 2013).

[8] Lee, P. (2011). Design Research: What Is It and Why Do It? http://thereboot.org/blog/2011/02/06/designresearch-what-is-it-and-why-do-it/ (Access date: 9 January 2012). 
[9] Malamed, C. (n.d.) Learning technology trends to watch in 2012. http://theelearningcoach.com/elearning20/learning-technology-trends-for-2012/ (Access date: 12 January 2012).

[10] Pocatilu, P. (2010). Developing mobile learning applications for android using web services. Informatica Economica, 14(3), 106-115.

[11] Pocatilu, P. (2011). Implementation of Distributed MLearning Applications Using WF and WCF. Informatica Economica, 11(1), 122-131.

[12] Richardson, L. S. (2010). Meme-ingful Education. http://designmind.frogdesign.com/blog/meme-ingfuleducation.html (Access date: 12 January 2012).

[13] Anywhere Software (2012). Basic for Android Android Programming with Gui Designer. http://www.basic4ppc.com/android/why.html (Access date: 9 May 2012).

[14] Erturk, E. (2009). International technology transfer: the case of free computer software. International Academy of Business and Public Administration Disciplines (IABPAD) 2009 Winter Conference in Orlando, Florida. http://repository.digitalnz.org/system/uploads/record/attach ment/596/international_technology_transfer_the_case_of _free_computer_software.pdf (Access date: 27 January 2013).

[15] Holstein, B. (2011). Speed delivery of Android devices and applications with model-driven development. http://www.ibm.com/developerworks/rational/library/mode 1-driven-development-speed-delivery/

(Access date: 9 May 2012).

[16] Schulz, T. (2012). FreeHAL is a self-learning conversation simulator. https://code.google.com/p/freehal/ (Access date: 9 May 2012).

[17] Vanel, J. (2012). EulerGUI Manual. http://eulergui.sourceforge.net/documentation.html (Access date: 9 May 2012).

[18] Bhat, A. (2011). Microlearning: A Paradigm Shift in the Way We Learn. Instructional Design. http://www.upsidelearning.com/blog/index.php/2011/01/2 8/microlearning-a-paradigm-shift-in-the-way-we-learn/ (Access date: 9 May 2012).

[19] Cisco Systems. (2013). The Cisco Learning Network. https://learningnetwork.cisco.com/community/learning_ce nter/m-learning (Access date: 27 January 2013). 